\title{
Sum rules and a coupled cluster formulation of linear response theory
}

DOI:

10.1007/BFb0037568

\section{Document Version}

Accepted author manuscript

Link to publication record in Manchester Research Explorer

\section{Citation for published version (APA):}

Bishop, RF. (1984). Sum rules and a coupled cluster formulation of linear response theory. In H. Kümmel, \& M. L. Ristig (Eds.), Recent Progress in Many-Body Theories (pp. 310-318). (Series on Lecture Notes in Physics; Vol. 198). Springer Nature. https://doi.org/10.1007/BFb0037568

\section{Published in:}

Recent Progress in Many-Body Theories

\section{Citing this paper}

Please note that where the full-text provided on Manchester Research Explorer is the Author Accepted Manuscript or Proof version this may differ from the final Published version. If citing, it is advised that you check and use the publisher's definitive version.

\section{General rights}

Copyright and moral rights for the publications made accessible in the Research Explorer are retained by the authors and/or other copyright owners and it is a condition of accessing publications that users recognise and abide by the legal requirements associated with these rights.

\section{Takedown policy}

If you believe that this document breaches copyright please refer to the University of Manchester's Takedown Procedures [http://man.ac.uk/04Y6Bo] or contact uml.scholarlycommunications@manchester.ac.uk providing relevant details, so we can investigate your claim.

\section{OPEN ACCESS}




\title{
SUM RULES AND A COUPLED CLUSTER FORMULATION
}

\section{OF LINEAR RESPONSE THEORY}

\author{
R.F. Bishop \\ Department of Mathematics \\ University of Manchester Institute of Science and Technology \\ P.0. Box 88, Manchester M60 1QD, England
}

\section{INTRODUCTION}

It is my intention here to describe the recent developments of the coupled-cluster formulation of quantum many-body theory in which we have succeeded in imbedding the we11-known theory of linear response within this formalism, and have shown how a new hierarchy of very useful sum rules thereby emerges. It will transpire in so doing that the new formalism also provides a very convenient bridge between the previously somewhat separate (although, of course, related) coupled-cluster formalisms for the ground and excited states respectively of the many-body system under consideration.

\section{COUPLED-CLUSTER DECOMPOSITION OF THE SCHRÖDINGER EQUATION}

A very brief outline is first presented of such of the main elements of the coupled-cluster formalism as are needed here.

\subsection{Ground-state formalism}

The usual starting-point for the ground-state (g.s.) coupled-cluster formalism (CCF) is an exact re-expression of the many-body g.s. Schrödinger equation in terms of a set of non-linear coupled equations for the so-called correlation amplitudes.

Purely for ease of present exposition the discussion is given here wholly in terms of infinite, homogenous systems of bosons, for which the coupled-cluster ansatz for the exact g.s. wavefunction $|\psi\rangle$ is given as,

$$
|\psi\rangle=e^{S}|\Phi\rangle ; S=\sum_{n=1}^{N} S_{n},
$$

in terms of an $\mathrm{N}$-body model or reference g.S. $|\Phi\rangle$ which is taken to be a single-state (usually zero-momentum) condensate,

$$
|\Phi\rangle=(N !)^{-\frac{1}{2}}\left(b_{0}^{+}\right)^{N}|0\rangle \text {, }
$$

where $|0\rangle$ is the vacuum state. In terms of a complete set of boson creation operators $b_{\alpha}^{\dagger}$, which create the complete orthonormal single-particle (s.p.) basis $|\alpha\rangle$ when acting on the vacuum, the correlation operators $S_{n}$, which excite $n$ particle-hole pairs from this condensate, may be written as

$$
s_{n}=(n !)^{-1} \sum_{\rho_{1}} \cdots \rho_{n} b_{\rho_{1}}^{+} \cdots b_{\rho_{n}}^{+}\left(N^{-\frac{1}{2}} b_{0}\right)^{n} s_{n}\left(\rho_{1} \cdots \rho_{n}\right)
$$


where the labels $\rho_{1} \cdots \rho_{n}$ indicate non-condensate s.p. states, thereby displaying the linked-cluster aspect of the original $\mathrm{e}^{\mathrm{S}}$ ansatz of Eq. (1). The derivation of the g.s. coupled-cluster equations is now formally performed in two simple steps. The g.s. Schrödinger equation, with energy eigenvalue $E$ is first pre-multiplied by the operator $e^{-S}$,

$$
e^{-S_{H e} S_{\mid \Phi>}}=E \mid \Phi>,
$$

which may be considered as a purely formal step to eliminate some "unlinked" terms from the outset that otherwise need to be eliminated later. The scalar product is then finally taken of Eq. (4) either with the model state $|\Phi\rangle$ or with the states

$$
b_{\rho_{I}}^{\dagger} \cdots b_{\rho_{n}}^{+}\left(N^{-\frac{1}{2}} b_{0}\right)^{n} \mid \Phi>\text {. }
$$

Clearly, when $\rho_{1} \cdots \rho_{n}$ run over all (non-condensate) s.p. states of the complete s.p. basis, and when $n$ runs from 1 to $N$, the vectors $|\Phi\rangle$ and (5) span the entire $\mathrm{N}$-body Hilbert space. Thus the set of equations

$$
\begin{aligned}
\left\langle\Phi\left|e^{-S_{H e} S}\right| \Phi\right\rangle & =E, \\
\left\langle\Phi\left|\left(N^{-\frac{1}{2}} b_{0}^{+}\right)^{n_{b}}{ }_{\rho_{n}} \cdots b_{\rho_{1}} e^{-S_{H e} S}\right| \Phi\right\rangle & =0,
\end{aligned}
$$

which are the g.s. coupled-cluster equations, are hence fully equivalent to the $\mathrm{N}$-body Schrödinger equation. They are a coupled set of nonlinear equations for the matrix elements (or subsystem amplitudes) $S_{n}\left(\rho_{1} \cdots \rho_{n}\right)$ of the correlation operators $S_{n}$. In order to be useful in practice one has to truncate this hierarchy and, for example, the "natural" truncation of the so-called SUBn scheme, wherein each of the amplitudes $s_{i}$ is set to zero for $i>n$ and the remaining $n$ coupled equations are solved for the amplitudes $s_{i}$ with $i \leq n$, has by now been thoroughly investigated. Thus it is by now well known that the numerical solution of appropriately truncated subsets of the equations (6) has lead to excellent quantitative g.s. results for systems as diverse as closed-she1l atomic nuclei, the one-component coulomb plasma, and even quite complex systems from the realm of quantum chemistry.

For further details of a formulation of the g.s. formalism that perhaps best stresses its physical content, the interested reader is referred to the article by Lührmann. ${ }^{1}$ A full review has also been given ${ }^{2}$ in the context of applications to nuclear physics, and hence where interest is particularly focussed on short-range correlations. For the particular problems inherent to the cases of long-range interactions and longrange correlations, the reader is also directed to the essentially self-contained articles by Lührmann and the present author, ${ }^{3,4}$ which deal with the one-component Coulomb plasma (or 'jellium') in SUB2 approximation.

\subsection{Excited-state formalism}

The g.s. formalism already described presumably may be employed not only for the g.s. but also for those states (with the same imposed symmetry as the g.s.) that have non-zero overlap with the model state $\mid \Phi>$. (We note that Eq. (1) automatically imp- 
lies a normalisation $\langle\Phi \mid \psi\rangle=1$.) Thus restricting ourselves to excited states $\left|\Psi_{\ell}\right\rangle$ which are orthogonal to both $|\Phi\rangle$ and $|\psi\rangle$, Emrich ${ }^{5}$ has recently shown that an appropriate choice of CCF wavefunction is,

$$
\begin{aligned}
& \left|\Psi_{\ell}\right\rangle=S^{(l)} e^{S}|\Phi\rangle, S^{(l)}=\sum_{n=1}^{N} S_{n}^{(l)}, \\
& S_{n}^{(l)}=(n !)^{-1} \sum_{\rho_{1} \cdots \rho_{n}} b_{\rho_{1}}^{+} \cdots b_{\rho_{n}}^{+}\left(N^{-\frac{1}{2}} b_{0}\right)^{n} S_{n}^{(l)}\left(\rho_{1} \cdots \rho_{n}\right) .
\end{aligned}
$$

Each non-zero vector $S_{n}^{(l)} \mid \Phi>$ is assumed to have a non-zero overlap with $\mid \Psi_{l}>$. If the excited state is a momentum eigenstate with eigenvalue $\vec{q}$ and we choose to work in a plane-wave s.p. basis, this implies that the s.p. momenta $\rho_{1} \cdots \rho_{n}$ in Eq. (7) must add to $\vec{q}$, whereas in the g.s. Eq. (3) they must add to zero.

The formal derivation of the excited-state (e.s.) coupled-cluster equations is now again easily performed. The e.s. Schrödinger equation, with energy eigenvalue $E_{\ell} \equiv$ $E+\omega$ (i.e. with excitation energy, $\omega$ ), is first combined with its g.s. counterpart, to give

$$
\left[H, S^{(l)}\right]\left|\psi>=\omega^{(l)}\right| \psi>.
$$

A similar procedure as in the g.s. case above then leads to the e.s. counterpart of Eq. (6) as

$$
\left.\langle\Phi|\left(N^{-\frac{1}{2}} b_{0}^{+}\right)^{n_{1}} b_{\rho_{n}} \cdots b_{\rho_{1}} e^{-S_{[H, S}(l)}\right] e^{S}|\Phi\rangle=\omega S_{n}^{(l)}\left(\rho_{1} \cdots \rho_{n}\right) .
$$

Equations (9) are thus the linked e.s. coupled-cluster equations, and we note that they take the form of a coupled set of linear eigenvalue equations for the e.s. subsystem amplitudes, with the same (excitation energy) eigenvalue $w$ in each equation. In each equation, the g.s. solution is assumed already known so that the g.s. correlation amplitudes are input to Eqs. (9).

Just as in the g.s. case, the e.s. Eqs. (9) also have to be truncated to be usefur in practice. As an obvious extension of the g.s. SUBn scheme, for example, we mention the $\operatorname{SUB}(m, n)$ scheme where the $n$ lowest equations of Eq. (6) and the $m$ lowest equations of Eq. (9) are solved in the approximation that the operators $S_{m+k}^{(l)}$ and $S_{n+k}$ are set to zero for all $k \geq 1$. However, one obvious point that arises immediately is the choice of "compatible" $(m, n)$ pairs. For example, one would like to know a priori whether for a given $n$, higher values of $m$ in the SUB $(m, n)$ scheme necessarily lead to a "better" approximation. Such questions are difficult to answer without further information, and it is in this sense that the theory of linear response to be presented, provides a bridge between the otherwise essentially disparate g.s. and e.s. formalisms already described.

\section{LINEAR RESPONSE AND GENERAL SUM RULES}

Let us now consider the response of the system to the addition of a small perturbation $\lambda V$ to the hamiltonian $H$, by expanding the g.s. energy and wavefunction in powers of the coupling parameter $\lambda$, 


$$
\begin{aligned}
H^{\prime} & =H+\lambda V, \\
E^{\prime} & =E+\lambda E(1)+\lambda^{2} E^{(2)}+\cdots, \\
\mid \Psi^{\prime}> & =|\Psi>+\lambda| \Psi(1)>+\lambda^{2} \mid \Psi\left({ }^{2}\right)>+\cdots .
\end{aligned}
$$

One possible means of progressing within the CCF at this point is to define $e^{S^{\prime}} \mid \Phi>$ as the perturbed g.5. $\left|\Psi^{\prime}\right\rangle$, and to use Eqs. (10) to determine the perturbed correlation operator $\mathrm{S}^{\prime}$, as has recently been discussed by Arponen. ${ }^{6}$ As an alternative however, and guided by the usual derivation of sum rules, we make contact at this point with (at least part of) the excitation spectrum $\left|\Psi_{\ell}\right\rangle$ of the unperturbed hamiltonian $H$ by expanding the first-order correction to the g.s. Wavefunction as

$$
\left|\Psi^{(1)}\right\rangle=\sum_{\ell} g_{\ell}\left|\Psi_{\ell}\right\rangle ; H\left|\Psi_{\ell}\right\rangle=E_{\ell}\left|\Psi_{\ell}\right\rangle,
$$

where the coefficients $g_{\ell}$ are as yet unknown. We now restrict ourselves to firstorder changes in the g.s. wavefunction only (linear response theory), and also impose as further restrictions from the outset that the excited states $\left|\Psi_{l}\right\rangle$ entering the expansion (11) are orthogonal to the model condensate state $|\Phi\rangle$ (as in Sect. 2.2). Further, we restrict the ensuing discussion to perturbations $v$ such that the inner products of the vector $v|\Psi\rangle$ with both $|\Phi\rangle$ and $|\psi\rangle$ are zero. The standard analysis of linear response then readily shows both that the first-order energy change $E^{(1)}$ vanishes, and the further results,

$$
\begin{aligned}
& \sum_{\ell} \omega_{\ell} g_{\ell}\left|\Psi_{\ell}\right\rangle=-v|\Psi\rangle ; \\
& g_{\ell}=-\frac{1}{\omega_{\ell}} \frac{\langle\Psi \ell|v| \Psi\rangle}{\left\langle\Psi_{\ell}\right| \Psi_{\ell}} ; \\
& E^{(2)}=\sum_{\ell} g_{\ell} \frac{\left\langle\Psi|v| \Psi_{\ell}\right\rangle}{\langle\Psi \mid \Psi\rangle} .
\end{aligned}
$$

For future purposes it is also convenient to consider the "m th power" of the perturbed Schrödinger equation, namely

$$
(H+\lambda v)^{m}\left|\Psi^{\prime}\right\rangle=E^{\prime m}\left|\Psi^{\prime}\right\rangle \text {. }
$$

It is then straightforward to use Eqs. (10) and (11) in expanding Eq. (15) to first order in $\lambda$, to show that for any integral $\mathrm{m}$,

$$
\sum_{\ell} \omega_{\ell}^{m} g_{\ell}\left|\Psi_{\ell}\right\rangle=-v_{(m)}|\psi\rangle,
$$

where $v_{(m)}$ is a nested commutator, defined iteratively as

$$
v_{(1)}=v ; v_{(m)}=\left[H, v_{(m-1)}\right], m>1 .
$$

Equations (12) and (16) now constitute the basis for our general hierarchies of sum rules. Thus, by taking their inner products with the states given in Eq. (5), after a prior pre-multiplication by $\mathrm{e}^{-\mathrm{S}}$, gives the sum rules

$$
\sum_{l} \omega_{\ell}^{m} g_{\ell} S_{n}^{(l)}\left(\rho_{1} \cdots \rho_{n}\right)=-\left\langle\Phi\left|\left(N^{-\frac{1}{2}} b_{0}^{\dagger}\right)^{n} b_{\rho_{n}} \cdots b_{\rho_{1}} e^{-S_{v}}(m) e^{S}\right| \Phi\right\rangle \equiv-F_{m n}\left(\rho_{1} \cdots \rho_{n}\right) .
$$


We note in particular that the sum rules (18) relate the excitation energies and correlation amplitudes on the one hand with the ground-state correlations on the other. Equation (14) [which together with Eq. (13) is just second-order perturbation theory for the g.s. energy] may also be regarded as a kind of zeroth order sum rule.

\section{RELATION TO SUM RULES FOR THE STRUCTURE FUNCTION}

A particularly important application of the above analysis, motivated by the res trictions discussed below Eq. (11), now follows from the choice,

$$
\begin{aligned}
& v=\frac{1}{2}\left(\rho_{\vec{q}}+\rho_{\vec{q}}^{+}\right) \equiv v^{+} ; \quad(q \neq 0) . \\
& \rho_{\vec{q}} \equiv N^{-\frac{1}{2}} \sum_{\vec{k}} b_{\vec{k}}^{+} b_{\vec{k}+\vec{q}} \equiv \rho^{\dagger} \vec{q}
\end{aligned}
$$

The operator $\rho_{\vec{g}}^{+}$creates a density fluctuation with momentum $\vec{q}$, and the perturbation $v$ thus destroys the translational invariance of the original hamiltonian. Working again in a momentum-eigenstate (i.e., plane-wave) s.p. basis, with $|\Phi\rangle$ the zeromomentum condensate, it is clear from Eq. (13) that the only excited states of interest, namely those that carry non-zero weight $g_{\ell}$ in Eq. (11), are momentum eigenstates with eigenvalue $\vec{q}$ or $-\vec{q}$. Hence to obtain non-trivial results from $\mathrm{Eq}$. (18), the momenta $\rho_{1} \cdots \rho_{n}$ must also add either to $\vec{q}$ or $-\vec{q}$, and in the following we assume they sum to $\vec{q}$. In the simplest case, $m=1$, the right-hand side of Eq. (18) can now be evaluated with the perturbation of Eq. (19), to give,

$$
F_{11}(\vec{q})=\frac{1}{2}\left[1+S_{2}(q)\right] ; S_{2}(q) \equiv S_{2}(\vec{q},-\vec{q}) \text {, }
$$

and the other functions $F_{l n}$ with $n>1$ can be similarly evaluated in terms of the g.s. subsystem amplitudes $S_{n}$. (Note that in the plane-wave s.p. basis, $S_{1} \equiv 0$ by momentum conservation.) In this way, one can show for example that the lowest order $(n=1)$ sum rules derived from Eq. (18) with $m=1,2$ are respectively given as

$$
\begin{aligned}
& \sum_{\ell} \omega_{\ell} g_{\ell} s_{1}^{(\ell)}(\vec{q})=-F_{11}(\vec{q}), \\
& \sum_{\ell} \omega_{\ell}^{2} g_{\ell} s_{1}^{(\ell)}(\vec{q})=-\frac{\hbar^{2} q^{2}}{2 m}\left\{1-F_{11}(\vec{q})\right\},
\end{aligned}
$$

and the other $(m, n)$ sum rules can be similarly evaluated.

In the limit of vanishing momentum transfer, the energy shift due to the perturbation of Eq. (19) can also be calculated macroscopically in the usual well-known fashion, to give the "compressibility sum rule" for the dynamic structure function $T(q, w)$,

$$
T(q, \omega) \equiv \sum_{\ell} \frac{\left\langle\left.\Psi\right|_{\vec{q}}\left|\Psi_{\ell}><\Psi_{\ell}\right| \rho_{\vec{q}}^{\dagger} \mid \psi\right\rangle}{\left\langle\Psi\left|\Psi><\Psi_{\ell}\right| \Psi_{\ell}\right.} \delta\left(\omega-\omega_{\ell}\right),
$$

which can be expressed in our CCF language and in the usual way as

$$
\lim _{q \rightarrow 0} 2 \sum_{\ell} g_{\ell} \frac{\left\langle\Psi|\rho \vec{q}| \Psi \ell^{\rangle}\right.}{\langle\Psi \mid \Psi\rangle}=-\frac{1}{2 m c^{2}} \Longleftrightarrow \lim _{q \rightarrow 0} \int_{0}^{\infty} d \omega \omega^{-1} T(q, \omega)=\frac{1}{2 m c^{2}},
$$


in terms of the first-sound velocity, c. Two other well-known sum rules for $T(q, w)$, namely the "static sum rule" for the static function $T(q)$ and the "f-sum rule", can also easily be derived by taking the inner product with the vector $v / \psi>$ of Eq. (16) with $n=1$ and $m=1,2$ respectively:

$$
\begin{aligned}
& 2 \sum_{\ell} \omega_{\ell} g_{\ell} \frac{\left\langle\Psi\left|\rho_{q}\right| \Psi_{\ell}\right.}{\langle\Psi \mid \psi\rangle}=-T(q) \Longleftrightarrow \int_{0}^{\infty} \mathrm{d} \omega T(q, \omega)=T(q) ; \\
& 2 \sum_{\ell} \omega_{\ell}^{2} g_{\ell} \frac{\left\langle\Psi\left|\rho_{q}\right| \Psi_{\ell}\right\rangle}{\langle\Psi \mid \Psi\rangle}=-\frac{\hbar^{2} q^{2}}{2 m} \Longleftrightarrow \int_{0}^{\infty} \mathrm{d} \omega \omega T(q, \omega)=\frac{\hbar^{2} q^{2}}{2 m} .
\end{aligned}
$$

In order to compare Eqs. (26), (27) with Eqs. (22), (23) it is most useful to re-

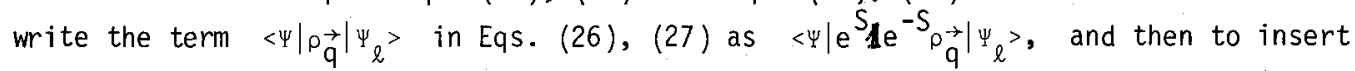
the following identity in the $\mathrm{N}$-particle Hilbert space for the unit operator so indicated,

$$
\mathbb{1} \equiv \mid \Phi>\left\langle\Phi\left|+\sum_{n} \frac{1}{n !} \sum_{\rho_{1}} \sum_{\rho_{n}} b_{\rho_{1}}^{+} \cdots b_{\rho_{n}}^{+}\left(N^{-\frac{1}{2}} b_{0}\right)^{n}\right| \Phi><\Phi\right|\left(N^{-\frac{1}{2}} b_{0}^{+}\right)^{n_{1}} b_{\rho_{n}} \cdots b_{\rho_{1}} .
$$

In this way it is easy to see that the first term on the right-hand side of Eq. (28) when inserted as indicated in the left-hand sides of Eqs. (26) and (27) yields just exactly the left-hand sides of Eqs. (22) and (23) respectively. From the remaining term on the right-hand side of Eq. (28) we get for larger $n$ a very complex and non linear dependence on the g.s. correlation amplitudes $S_{n}$ but with each term linear in the e.s. amplitudes $S_{n}^{(l)}$. In this way one shows that the sets of sum rules (18) with $m=1,2$ respectively (and in each case for all $n$ ) correspond to the sum rules of Eqs. (26) and (27) for $T(q, w)$. The same is clearly also true for the (perhaps less familiar) higher $(m>2)$ sum rules which represent the higher moments of the structure function $T(q, w)$. In each case, for a given moment (or index $\mathrm{m}$ ), our system of sum rules constitutes a cluster decomposition (in index $n$ ) of the corresponding sum rule for the dynamic structure function, into sub-sum-rules.

\subsection{One-state approximation}

As a preliminary indication of the usefulness of the new sum rules, we now assume that a single excited state exhausts the sum rules -- the so-called one-state approximation. In this case, division of Eq. (23) by Eq. (22) yields the relation,

$$
\omega \rightarrow \omega(q)=\frac{\hbar^{2} q^{2}}{2 m} \frac{1-S_{2}(q)}{1+S_{2}(q)},
$$

where use has also been made of Eq. (21). In order to make use of Eq. (25) to make further progress we now need to evaluate the left-hand side of Eq. (25) by the same procedure as already indicated between Eqs. (27) and (28). To make the calculation tractable we also work in the random-phase approximation (RPA) as the obvious approximation scheme, and which is characterised by neglecting all contributions which depend on momenta other than $\pm \vec{q}$, and by setting $S_{j}=0$ for $i \geq 3$. In this approximation, 
the second term on the right-hand side of Eq. (28) gains a non-zero contribution only from the $n=2$ term in the sum. The remaining factors can now be evaluated using similar techniques of inserting appropriate unit operators. Space considerations preclude a detailed analysis, and we quote only the final result (true only in the RPA):

$$
\left\langle\Psi\left|\rho_{q}\right| \Psi_{\ell}>/\langle\Psi \mid \psi\rangle \simeq S_{1}^{(l)}(q)[]-S_{2}(q)\right]^{-1} \text {. }
$$

Inserting Eq. (30) into Eq. (25), and combining the resulting equation with Eqs. (21) (23), yields the further results in the one-state approximation,

$$
\lim _{q \rightarrow 0} S_{2}(q)=-1+\frac{\hbar q}{m c} ; \lim _{q \rightarrow 0} \omega(q)=\hbar c q \text {. }
$$

Equation (31) shows that this simple approximation leads to the universal existence of a low-momentum phonon branch of the excitation spectrum. Furthermore, the Bose equation for $S_{2}(q)$ from the coupled-cluster equations $(6)$ is given in the RPA by ${ }^{4}$

$$
\frac{\hbar^{2} q^{2}}{m} S_{2}(q)+N V(q)\left[1+S_{2}(q)\right]^{2}=0 \text {, }
$$

in terms of the two-body potential $V$. Use of Eq. (31) in Eq. (32) then also yields the well-known relation for the sound velocity,

$$
c=[N V(0) / m]^{\frac{1}{2}} \text {. }
$$

The static structure function $T(q)$ can also be evaluated in the RPA from Eq. (26) by use of Eq. (30). Comparing the resulting equation with Eq. (22), evaluating both in the one-state approximation, and using also Eq. (21), then yields the result valid in the RPA,

$$
T(q)=\left[1+S_{2}(q)\right] /\left[1-S_{2}(q)\right] .
$$

A comparison of Eqs. (29) and (34) finally yields the wel1-known Bijl-Feynman relation between the static structure function and the excitation spectrum,

$$
T(q) \omega(q) \simeq \frac{\hbar^{2} q^{2}}{2 m} \text {. }
$$

It is of some interest to note that the only approximation involved in deriving Eq. (29) is the one-state approximation, whereas Eqs. (34) and (35) have also involved the RPA. We note however that making the one-state approximation also in Eq. (27), which we have so far not used, and comparing with Eq. (26), yields Eq. (35) directly but now without using the RPA. It must be carefully noted that an evaluation of $T(q)$ in a different approximation to RPA will therefore not be wholly compatible with the one-state approximation expressed by Eq. (35). We finally note that al though the onestate approximation is compatible with the RPA at the level of Eqs. (22), (23), (26) and (27), it is certainly not exact. When higher sum rules (18) (e.g. $m=3, n=1$ ) are also simultaneously considered, inconsistencies start to arise.

\section{FINAL REMARKS}

We have seen how to derive a set of exact sum rules in the CCF which connect pro- 
perties of the excitation spectrum characterised by $\left|\Psi_{\ell}\right\rangle$ and $S_{n}^{(l)}$, with ground-state properties characterised by $\mid \psi>$ and $S_{n}$. Our new double manifold (in indices $m, n$ ) of sub-sum-rules (18) represents a cluster decomposition of the usual single manifold of sum rules for the energy-weighted moments of the dynamic structure function, and thereby is able to provide much more detailed information about many-body systems than the latter. The new formalism should also be useful in applications to systems as liquid ${ }^{4}$ He where the first step would probably be to use it to determine the g.s. correlation function $S_{2}(q)$ from the experimental spectrum $w(q)$. In this way one could investigate the use of excited-state properties to determine the correlations present in the g.s., which have otherwise proven notoriously difficult to unravel. It is also possible that our new sub-sum-rules will enable the CCF and the alternative many-body moment methods ${ }^{7}$ mutually to illuminate one another.

We have seen how even the simplest approximation for these new sum rules of assurijng that the excitation spectrum consists of a single state (which therefore exhausts the sum rules), leads to a phonon spectrum in the long-wavelength limit, and also to the Bijl-Feynman relation when the RPA is further assumed. We note that these results, which can also be derived from the structure function sum rules in the same one-state approximation, were derived using the $(m, n)$ sub-sum-rules (18) for the case $n=1$ only. This is not surprising since phonons are just (collective) coherent superpositions of one-particle/one-hole excitations, and which are therefore fully described by $s_{1}^{(l)}$ and which hence correspond to the case $n=1$. only. Extensions of the use of the subsum-rules (18) to the case $n>1$ is likely also to be of some interest. How to improve upon the one-state approximation, perhaps with the aim of making arbitrarily many of the $(m, n)$ sub-sum-rules (18) compatible with each other (and with the corresponding sum rules for the structure function), also remains an open question of considerable importance. In this same spirit, the difficulty of really proving that phonons become exact eigenstates in the low-q limit has also been emphasised in other contexts by Feenberg. 8

Finally, we hope that the sum rule formalism developed here may open the door to a wider range of applications of the CCF, which has hitherto largely been used only to solve (approximately) the g.s. and e.s. Schrödinger equation with given many -body hamiltonian. In particular, we believe that the sum rules are likely to prove useful in investigating the compatibility between otherwise disparate approximations in the essentially distinct g.s. and e.s. CCF methods. More generally, it seems clear that in principle, all systems to which the CCF has already very successfully been applied, are worth studying anew with these newly-developed tools.

\section{References}

1 K.H. Lührmann, Ann.Phys. (NY) 103 (1977) 253.

2 H. Kümmel, K.H. Lührmann and J.G. Zabolitzky, Phys. Reports 36C (1978) 1.

3 R.F. Bishop and K.H. Lührmann, Phys.Rev. B 17 (1978) 3757. 
4 R.F. Bishop and K.H. Lührmann, Phys.Rev. B 26 (1982) 5523.

5 K. Emrich, Nucl.Phys. A351 (1981) 379, 397.

$6 \mathrm{~J}$. Arponen, University of Helsinki preprint HU-TFT-81-41 (1981).

7 B.J. Dalton, S.M. Grimes, J.P. Vary and S.A. Williams (Eds.), "Moment Methods in Many-Fermion Systems," Plenum, New York (1980).

8 E. Feenberg, "Theory of Quantum Liquids," Academic Press, New York, (1969), p.72. 